\title{
EARLY VISUAL DEVELOPMENT: DIFFERENTIAL FUNCTIONING OF PARVOCELLULAR AND MAGNOCELLULAR PATHWAYS
}

\author{
JANETTE ATKINSON \\ Cambridge
}

\section{INTRODUCTION}

The idea of two visual systems arose out of comparative studies of vision across different species. ${ }^{1}$ It was suggested that subcortical systems control orienting responses which define crudely 'where' an object is located and trigger foveation to that location, while cortical mechanisms are used to define 'what' is actually in the foveated area. Using this idea, Bronson ${ }^{2}$ put forward a model of human development suggesting that newborn vision is totally subcortically controlled with the cortex maturing at around two months postnatally. Atkinson, ${ }^{3}$ using both electrophysiological and behavioural data from infant studies, put forward a modified 'two visual systems' model of development. The newborn visual system is 'largely' subcortically controlled, with different cortical mechanisms starting to function at different times postnatally.

In this paper the ideas behind this theory are updated in the light of recent research on infants. Two theoretical models of adult vision are used to make analogies with infant vision. The first is based largely on primate lesion studies, where dissociations relating to different lesions have been noted. A 'where' and a 'what' system have been defined solely within the cortical pathways. The initial evidence for selective processing of visual information in primate extrastriate cortex was obtained by Zeki and his co-workers. They defined an area selective for the direction of stimulus motion which has since been called V 5 or MT. ${ }^{4}$ In other studies Zeki ${ }^{5,6}$ identified a colour specific area, V4. Ungerleider and Mishkin ${ }^{7}$ suggested that these two streams are associated with different visual capacities-a largely parietal module is involved with localising objects within a spatial array, while those involving the temporal lobe contain mechanisms tuned to 'what' aspects such as form and colour. Clinical observations of patients

Visual Development Unit, Craik Laboratory, University of Cambridge.

Correspondence to: Visual Development Unit, Department of Experimental Psychology, University of Cambridge, Downing Street, Cambridge CB2 3EB. with specific focal lesions have shown a dissociation between loss of position or movement perception and deficits of object recognition. ${ }^{8,9,10,11}$

The second model of adult visual processing arises out of the first and is based on two types of anatomically and morphologically distinct ganglion cells (parvocellular and magnocellular). The two streams are separate at the subcortical levels with parvocellular and magnocellular neurons projecting to different regions of primary visual cortex, V1. ${ }^{12,13,14}$ In V1 the superficial layers contain an array of dot-like patches of cytochrome oxidase rich tissue called 'blobs ${ }^{15,16,17}$ which have a largely parvocellular input. Blobs seem to be specific for colour while interblobs are important for orientation. Within V2 there are regions of high and low cytochrome oxidase-rich activity. Those that are high are in thin and thick stripes with neurons in the thin stripes projecting to V4 while those in the thick stripes project to V5. A parvocellular based system subserves detailed form vision and colour while the magnocellular system subserves movement perception and some aspects of stereoscopic vision. Anatomical and physiological findings allow identification of populations of neurons specific for detection of each visual attribute within these cortical streams. Information for different attributes within these cortical streams appears to be segregated at many different levels of the cortex. . $^{14,18,19}$

Comparisons have been made between psychophysical data on adults and the functioning of the parvocellularly based and magnocellularly based pathways. Similar comparisons are made below when looking at the development of vision in human infants over the first six months postnatally. As yet there is little anatomical or morphological data on the relative time course of development of these two subsystems in human infants (only Hickey ${ }^{20}$ ) and so the model is based on behavioural and visual evoked potential measures to delineate the developmental time course of various cortical streams, each subserving different aspects of spatial vision. Development postnatally is considered under two broad headings: 
1. Development of selectively tuned pools of neurons for visual processing of contrast, orientation, colour, direction of motion, texture, size and depth.

2. Development of eye movement control mechanisms necessary for sampling the visual world and used in shifting visual attention from one object to another.

\section{DEVELOPMENT OF NEURONS SPECIFICALLY TUNED TO DIFFERENT ATTRIBUTES OF SPATIAL VISION}

\subsection{Acuity and contrast sensitivity}

The most basic visual information concerning identification of an object must involve measures of acuity and contrast sensitivity. Although there is some debate as to the precise values of newborn acuity, there is general agreement that a very rapid improvement in analysis of spatial detail, reflected in changes in acuity and contrast sensitivity, takes place between birth and six months of age. Previous reviews have considered the relative role of photoreceptors, retinal neural mechanisms and post retinal mechanisms in limiting acuity. ${ }^{21,22}$ While retinal development plays a major role in determining the limits on spatial information transmitted by the infant's visual system, post retinal neural immaturities must also play a part in explaining the newborn's poor contrast sensitivity and acuity (on average around $1.5 \mathrm{C}^{\circ}$ ) compared to that of the adult or indeed even a six month old infant (at least $6 \mathrm{C}^{\circ}$ ). We know that in the human visual cortex there is a massive increase in connectivity in the first six months of life $^{23}$ and presumably these new synapses are defining and refining stimuli to which cortical cells maximally respond. Using an approach where stimuli are 'designed' to elicit a response from cortical cells that have developed particular selective properties, we can track the time course of changing spatial abilities in the human infant. The responses are measured either as changes in behaviour or as visual evoked potentials.

\subsection{Development of neurons selective for orientation}

There is now general agreement that responses can be obtained from infants in the first few weeks of life indicating discrimination between a static grating pattern oriented at $45^{\circ}$ and one at $135^{\circ}$. Here infant control habituation procedures have been used..$^{24,25}$ An alternative approach is to record evoked potentials (VEPs) which can be identified by their consistent temporal relationship to the visual stimulus event. A steady state VEP (OR-VEP) can be elicited when a grating oriented at $45^{\circ}$ is replaced by one at $135^{\circ}$ in infants around six weeks postnatally. ${ }^{26} \mathrm{~A}$ statistically reliable response $e^{27}$ at the frequency of the orientation change (or at harmonics of this frequency) is evidence for orientation selective mechanisms. These mechanisms appear to have different orientation tuning and temporal tuning curves at different ages. At a rapid reversal rate between the two orientations (e.g. $8 \mathrm{rps}$ ), a significant response is found only in the second postnatal month. With slower alternations ( $3 \mathrm{rps}$ ), the median age for the response is three weeks. ${ }^{28}$ Interestingly, this rapid improvement in temporal sensitivity appears to be specific to the orientation response and not to non-orientational mechanisms. If the infant is shown a phase reversing (PR) grating, where the grating is in a constant orientation but the black and white lines are periodically interchanged at 3 rps or $8 \mathrm{rps}$, a significant PR-VEP can be recorded for infants from birth. This supports the view that the ORVEP is generated by a distinctive rapidly developing mechanism which is different from that responsible for the conventional PR response. Different ranges of temporal and spatial sensitivity have been found in the cell properties of the magnocellular and parvocellular pathways. ${ }^{29} \mathrm{It}$ is quite possible that the OR-VEP may reflect orientation selectivity in cortical cells forming part of the 'parvo stream' while the magno-stream is involved in transmitting non-oriented information. Alternatively, the nonoriented information, underlying the VEP, could be carried largely by subcortical pathways.

\subsection{Directional motion}

It is well established that young infants prefer moving to static visual displays. However, this in itself cannot be taken as evidence of the operation of true motion mechanisms. Any moving stimulus also produces a temporal modulation and it is well known that infants show a preference for full field flicker, i.e. temporal modulation without coherent motion. In general true motion detectors are in evidence if a differential response to different directions of motion can be demonstrated. To measure such mechanisms in infants we have used designer stimuli consisting of two dimensional random dot displays, which can have a particular direction of motion without the confounding presence of any dominant orientation component. In a similar way to the OR VEP technique above we can generate a VEP to a change in the direction of motion of a set of random dots. ${ }^{30,31}$ The first significant motion VEP for a velocity of $5 \% \mathrm{sec}$ appears at around two months of age, with onset for higher velocities occurring later. ${ }^{31}$ Parallel behavioural studies show once again that the velocity is a critical determinant in obtaining discrimination of relative motion at different ages. ${ }^{32}$

Many models of directional motion involve the comparison of spatially separated locations across a temporal delay. The maximum velocity at which the infant sees coherent motion (Vmax) will be determined by the spatial range and the minimum time interval at which relative motion detectors can operate. A change in the spatial range has been found to be the limiting factor determining changes in Vmax with increasing age from 12 weeks upwards. ${ }^{32}$

Many behavioural studies have concentrated on the lower rather than upper velocity threshold for detecting whether an object is moving at all (e.g. Aslin et al. ${ }^{33}$ ). The lowest detectable velocity (Vmin) or the corresponding minimum discrete displacement (Dmin) increases between eight and 20 weeks of age. However, most of these tasks do not require relative directional information, 
and the limit may be set by the simple spatial and temporal resolution of the infant's visual system at a particular age.

In conclusion it appears that both the upper and lower limits for discriminating direction motion increase with age, although true directional detectors have not as yet been demonstrated prior to eight weeks of age. The magnocellular stream running from ganglion cells to V1, V2, V3 and MT is related to information about direction. In the previous section we saw that some discrimination of orientations is possible at birth and it seems strange that information about direction of movement should not also be a built-in capability of the newborn, to provide fundamental information about 'where?'. Indeed the newborn does have a crude directional system already operating, as is evidenced by the optokinetic system giving optokinetic nystagmus $(\mathrm{OKN})$. In adults, both a cortical and subcortical pathway control OKN responses, whereas in newborn infants the occurrence of asymmetrical OKN for monocular viewing suggests that only the subcortical mechanisms are functioning. ${ }^{34,35}$ Thus it seems that the infant has at least two potential 'where' systems. One system is subcortical-the OKN mechanism (functional in the newborn), which operates maximally when the whole visual field is moving in a uniform direction. In adults this subcortical mechanism is normally suppressed by using the pursuit cortical mechanism to fixate and smoothly track a single object in the field of view. This later cortical system presumably operates within the magnocellularly based system and in developmental terms becomes functional later than the subcortical system.

Interestingly, several perceptual discriminations dependent on relative motion detection can be demonstrated in relatively young infants. For example, four month olds can distinguish between three dimensional forms which differ only in terms of 'structure from motion' cues in random dot displays. ${ }^{36}$ Many of the experiments on infants' abilities to make avoidance responses to looming objects (e.g. Yonas ${ }^{37}$ ) and to reach for nearby objects demonstrate how the infant integrates spatial and temporal information into a unified percept of an object in a given location.

\subsection{Binocularity}

As signals from the two eyes first interact at the cortex, any response dependent on detection of binocular correlation or disparity must be dependent on the operation of cortical rather than subcortical mechanisms. An example of such a response was first demonstrated in human infants using a sucking habituation paradigm. ${ }^{38}$ Such responses are first seen on average around three to four months postnatally. ${ }^{39,40,41,42,43,44,45}$ However, there are quite marked individual differences in functional onset, ranging from around eight weeks to 24 weeks. ${ }^{46}$ Binocular correlation and disparity detection appear to have the same onset time in individual infants, with significant VEP responses usually recorded a little before significant behavioural preferential looking responses. ${ }^{47}$ These studies indicate that the onset of functioning of cortical binocular mech- anisms is postnatal and such mechanisms cannot be used in any depth or distance judgements involved in spatial localisation made prior to three months of age. As the thick cytochrome oxidase staining stripes in V2, which receive input largely from the magnocellular system, are thought to be the predominant location of disparity selective neurons, it seems likely that development of functioning in this pathway takes place a few months postnatally in the case of human development.

Recently, attempts have been made to gauge the length of the human sensitive period for development of stereoscopic vision in a prospective study of infants who have become strabismic in the first six months of life. ${ }^{48,49}$ Measures of acuity, binocularity, and OKN responses have been made on this group both prior to surgery (on average at 15 months of age) and longitudinally up to four years of age. Although many of the strabismic children, who were initially alternating in fixation at the onset of their strabismus, showed some coarse disparity detection initially, very few of them showed any demonstrable stereopsis at four years of age. It would seem that a period of strabismus in the first year of life permanently disrupts the normal course of development of binocularity, and even good, relatively early surgical eye alignment cannot put that development back on its normal course. An additional finding was that most of these children did not develop severe amblyopia, suggesting that occlusion therapy together with eye alignment in the first year of life promotes development of almost normal acuity and contrast sensitivity. We do not know the exact level of compliance with occlusion therapy in this group, nor do we know whether successful occlusion therapy actually hinders development of stereopsis (while preventing amblyopia). However, given these unknowns, the cautious conclusion from these results would be support for the idea of separate critical periods for development of resolution acuity and stereopsis. This might be taken to suggest that the magnocellular system, underlying disparity detection, is more vulnerable and sensitive to aberrant environmental stimulation than the system underlying acuity development which is likely to be parvocellular in origin.

\section{DIFFERENTIAL ONSET OF FUNCTION IN PARVOCELLULAR AND MAGNOCELLULAR STREAMS}

Above, the findings on development of three types of cortical detectors have been considered. Each type is specific for coding information concerning a particular spatial attribute: orientation (necessary for shape analysis), directional movement (for analysing an object's trajectory), and correlation/disparity detection (for judging the relative depth or distance). Development of sensitivity to these attributes takes place largely postnatally and must depend on cortical rather than subcortical mechanisms.

Infants show sensitivity to changes of orientation before they show differential responses to the direction of movement and before they detect disparity change. Although not discussed here, infants in the first few weeks 
after birth show relatively sophisticated ability to detect differences based on colour alone, using isoluminant displays (e.g. Morrone et al. ${ }^{50}$ ). When we consider the adult models discussed in the Introduction, involving segregation of information in magnocellularly based and parvocellularly based streams, it would seem plausible to suggest that the parvo- become functional slightly before the magno-based stream in infant development. This discrepancy in functional onset times might lead to a dissociation in perception between colour plus form information and motion plus depth information. Such a dissociation has previously been alluded to in the literature by several investigators. For example, Bower ${ }^{51}$ suggested that infants up to around five months of age identify an object either in its static form or when moving along a trajectory-a static and moving object being seen as two different objects. Others (e.g. Baillargeon et al ${ }^{52}$ ) have noted that analysis of movement and local depth cues are linked. The infant sees two parts of a rod as one if they move along a common path behind a barrier. Two month olds tend to perceive the bar as in two parts if the display is static. Perhaps it is easier to integrate features if both are first rapidly analysed in specialised detectors. If one analysing stream is operating poorly (e.g. that for directional movement) then integration cannot take place across the two streams to perceive a unified object.

\section{DEVELOPMENT OF EYE MOVEMENT CONTROL SYSTEMS AND VISUAL ATTENTION}

Several different names have been given to tests which elicit eye movements to foveate objects of interest in the centre of the visual field. In the clinical literature it is called 'perimetry', in visual science it has been called 'foveation' and 'saccadic refixations', and in cognitive science it has been called 'shifts of attention' or 'selective attention'.

In infant development, several different mechanisms have been proposed to be responsible for such behaviour. Two such mechanisms are discussed below-the first is the saccadic localising processes for foveation (which are often accompanied in adults by focal attention), the second is mechanisms for segmentation (used for triggering the refixation mechanism).

\subsection{Saccadic refixation: localisation based on differences between target and background}

If a conspicuous large moving object is made to appear suddenly in the peripheral visual field, infants will often move their eyes to foveate the object centrally. Many studies indicate that this response is present but unreliable and inaccurate at birth, but becomes a robust response by three months of age. The size of the effective visual field has been measured using dynamic and static perimetry and found to increase with age. ${ }^{53,54}$ Of course, this improvement with age is likely to be due both to improvements in detection acuity and contrast sensitivity and to improvements in refixation processes to make the saccadic shifts.
We have tested such refixations in one to three month olds systematically varying the salience of the peripheral target, with an identical initially fixated central target either constantly visible or extinguished at the moment the peripheral stimulus appears. ${ }^{45,55}$ Even when an attempt is made to equate the peripheral targets for visibility above contrast threshold (a control procedure not normally made in clinical perimetry), one month olds tend to be a little slower in refixating, and often fail to refixate at all, if a competing central target is visible when the peripheral target appears.

\subsection{Segmentation: localisation based on texture detection}

Julesz $^{56}$ has proposed a theory of preattentive processing, defining classes of local features called 'textons' (such as edge segments and edge terminators) which enable rapid discrimination of texture patterns necessary for segmenting one object from another. Textons embody local phase relationships, i.e. the relative phase of the spatial frequency components making up the pattern elements. Relative phase information, together with amplitude or intensity, enables a complete specification of any pattern. Certain phase relationships will be 'special' in that they correspond to physical features of the outside world (e.g. the peaks-subtract phase relationship specifies a squarewave edge). A number of physiological models of relative phase detection in the brain have been proposed (e.g. Movshon et al. ${ }^{57}$ Pollen and Ronner ${ }^{58}$ ). In general all these models propose perception of configuration to be dependent on comparator detectors across spatial frequency channels. Such phase selectivity might, for example, be embodied in channels having even- and oddsymmetric receptive fields, and such mechanisms exist in the cortex rather than in the subcortex.

Applying these models, we have used tests of relative phase and texton discrimination to infer cortical functioning in young infants. It appears that one month olds are insensitive to changes in relative phase when viewing complex gratings made up of several superimposed spatial frequencies. ${ }^{59}$ Nor do they discriminate between texture patterns differing in the type of texton detectors proposed for adults. ${ }^{60}$ Three months olds have no difficulty with such discriminations.

One might imagine that an even simpler form of texture segmentation would be based on merely recognising areas containing identical single features, such as an area composed of similarly oriented short line segments. This segmentation process would take place after primary visual mechanisms have registered the simple stimulus properties of orientation, and would be used to define distinct surfaces, objects, and events in the visual world. In adults, the presence of orientation differences between line or edge segments making up two textures is very effective in determining visual segmentation..$^{61,62,63}$ Several models of cortical mechanisms responsible for these preattentive processes have been proposed (e.g. Sagi and Julesz ${ }^{64}$ Nothdurft ${ }^{65}$ ). Their principal component is local diffe- 
rencing operations, acting on the output of neurones which are visual filters for specific properties. The differencing function may depend on connections within the cortex providing inhibitory information between pools of neurons specifying differing orientations.

We have started to look for evidence of these cortical inhibitory mechanisms in infants by measuring their ability to orient towards a rectangular area in the visual field, the boundary being defined by a change in orientation of the line segments making up the display. The rectangle is presented to either the left or right of central fixation point. Using the forced choice preferential looking technique, we measure the infant's ability to detect the change of orientation within the rectangular patch compared to the background. What we have found is that this appears to be an impossible task for infants up to four months of age, and even at this age the response appears relatively weak. ${ }^{66,67}$ This relatively late onset of ability to segment on the basis of orientation suggests that other mechanisms for segmenting and localising objects in the visual world, such as contrast cues and differential motion, are more robust and useful in the first few months. Of course it may be that even in adult vision the primary segmenting mechanisms use crude intensity differences to parse the visual scene, and that these decisions are then confirmed by additional information provided by elaborate texture and phase comparators. It may also be that the orienting mechanism necessary for rapid detection of objects in the peripheral field does not use information processed in the cortex very effectively, but relies instead on crude information provided by the superior colliculus. Because of these possibilities we are now using a new paradigm, testing orientation segmentation in central vision, without the need for the peripheral orienting response. If infants younger than four months are indeed able to use local orientation differences to define the boundary of an object, although this discrimination is not used in orienting, then it would seem that the deficit in young infants is one of integration of information between registration and response systems, rather than a failure to register per se.

\section{ANALOGIES WITH VISUO- NEUROLOGICAL SYNDROMES INVOLVING DEFICITS OF SPATIAL VISION}

Blind-sight: A comparison can be made between the visual behaviour of newborn infants and adult humans and primates with deficits resulting from cortical lesions. In adult primates, if $\mathrm{V} 1$ is oblated then a difficulty in retrieving a small object (e.g. a raisin) is noted when the object is surrounded by a ring and is therefore competing for selective attention. ${ }^{68} \mathrm{~A}$ similar problem is seen in infants under six weeks of age in discriminating shapes if each shape is surrounded by an identical outer contour, and in recognising faces if the outer hair lines of both faces are made identical using swimming caps. ${ }^{69,70}$

'Blind-sight' patients (reviewed in Weiskrantz, ${ }^{71}$ Cowey and Stoerig ${ }^{72}$ ) can detect and discriminate flicker, orientation, wavelength (for large patches of colour) and simple shapes in their blind half field. However, they show no conscious awareness in these tasks. They have difficulty in matching complex patterns and in discriminating between different directions of motion. These difficulties result presumably from deficits of neurons for relative phase and relative motion discrimination and appear similar to the problems demonstrated by one month olds compared to three month olds in the experimental paradigms discussed above.

Balint's Syndrome: A second neurological condition, called 'Balint's Syndrome', is characterised by what has been called 'sticky fixation'. Patients have difficulty disengaging from a centrally fixated target and shifting their eyes to a new target of interest in the periphery. The problem is not one of 'neglect' or optic apraxia but rather concerns the co-ordination of mechanisms controlling saccadic eye movements and selective attention to provide accurate spatial localising. Balint's Syndrome involves bilateral lesions in parieto-occipital areas but may also involve the circuitry between the superior colliculus and parietal lobes for controlling shifts of attention (review by de $\operatorname{Renzi}^{73}$ ). Very similar behaviour to Balint's patients has been seen in primates with bilateral parietal damage, ${ }^{74}$ although Schiller has reported similar deficits with damage to the superior colliculus and frontal eye fields. ${ }^{75}$ Again the evidence for development of cortical streams would lend support to the idea that crude localising of single targets can be carried out by subcortical collicular mechanisms, while more elaborate selective processes, to shift attention from one object to another, require cortical executive control from the striate and extrastriate cortex.

Given our present knowledge from neuroscience, it is quite difficult to relate the deficits of attention involving eye movement control systems found in very young infants to differential development of cortical streams. We do not as yet know how the adult visual system integrates information across different cortical streams for form, colour and movement analyses, and between these streams and those controlling focal attention, so it would seem premature to surmise how these co-ordinating processes might develop. For the present we are left with observable changes in visual behaviour, measured as changes in eye movements, which must indicate a shift from subcortical to cortical executive control. Which particular pathways subserve these behavioural changes remains to be discovered.

\section{CONCLUSIONS}

In summary it appears that infants are born with operating discriminatory mechanisms linked to appropriate orienting responses which enable them to foveate crudely at birth (although morphologically the fovea and parafovea is not well differentiated). Newborn visual mechanisms are likely to be largely under subcortical control, given the results above concerning a lack of evidence for the operations of specialised detectors found in the cortex. However, there are a few exceptions-newborns have been found to discriminate large changes of orientation or slant 
(as in static low spatial frequency, high contrast grating patterns with orientations differing by $90^{\circ}$ ) which must depend on activity in cortical orientation-selective neurons. In the first few weeks postnatally many of the specialised detectors in the cortex start to operate to carry out discriminations of various visual attributes, such as the finer discriminations of orientation, colour and size. Separate cortical streams based on parvocellular and magnocellular distinctions have been hypothesised to underlie changes in visual capacity over the first six months of postnatal life. Parvocellular spatial representation systems for colour and form appear to be operational somewhat earlier postnatally than magnocellular-based systems for relative position, motion and depth. Integration of information within channels and across channels subserving different attributes may take at least four to five months to develop postnatally, as demonstrated in some tests of texture segmentation. Development of visual control mechanisms, linked to eye movements, have also been demonstrated over the first months postnatally. The changes that we can observe and measure not only involve a gradual shift from reflex subcortical control to cortex, they also suggest that mechanisms for form and object discrimination which are likely to involve parvocellularbased streams, carrying information to the temporal lobes, may be partially functional at birth or very soon postnatally. Mechanisms subserving disparity detection, fine discriminations of orientation and relative direction may take slightly longer to become tuned. These are likely to involve cortical streams originating in the magnocellular layers and carrying information to the parietal and frontal lobes for integration of information concerned with both location and form with those controlling eye movements and focal attention.

\section{REFERENCES}

1. Schneider GE: Two visual systems. Science 1969, 163: 895-902.

2. Bronson GW: The postnatal growth of visual capacity. Child Development 1974, 45: 873-90.

3. Atkinson J: Human visual development over the first six months of life. A review and a hypothesis. Human Neurobiology 1984, 3: 61-74.

4. Dubner R and Zeki SM: Response properties and receptive fields of cells in an anatomically defined region of the superior temporal sulcus. Brain Research 1971, 35: 528-32.

5. Zeki SM: Colour coding in rhesus monkey prestriate cortex. Brain Res 1973, 53: 422-7.

6. Zeki SM: Colour coding in the superior temporal sulcus of rhesus monkey visual cortex. Proc $R$ Soc London Series $B$ 1977, 197: 195-223.

7. Ungerleider LG and Mishkin M: Two cortical visual systems. In Ingle DG, Goodsale MA and Mansfield RJQ (Eds). Analysis of Visual Behavior 1982, 549-86. Cambridge MA: MIT Press.

8. Ratcliff G and Davies-Jones GAB: Defective visual localization in focal brain wounds. Brain 1972, 95: 49-60.

9. Damasio AR and Benton AL: Impairments of hand movements under visual guidance. Neurology 1979, 29: 170-8.

10. Pearlman AL, Birch J, Meadows JC: Cerebral color blindness: An acquired defect in hue discrimination. Ann Neurol 1979, 5: 253-61.
11. Zihl J, von Cramon D, Mai N: Selective disturbance of movement vision after bilateral brain damage. Brain 1983, 106: 313-40.

12. Livingstone MS and Hubel DH: Segregation of form, color, movement, and depth: anatomy, physiology, and perception. Science 1988, 240: 740-9.

13. Van Essen DC and Maunsell JHR: Hierarchical organization and functional streams in the visual cortex. Trends Neurosci 1983, 6: 370-5.

14. Maunsell JHR and Newsome WT: Visual processing in monkey extrastriate cortex. Ann Rev Neurosci 1987, 10: $3416-68$

15. Horton JC and Hubel DH: Cytochrome oxidase stain preferentially labels intersections of ocular dominance and vertical orientation columns in macaque striate cortex. Soc Neurosci Abstr 1980, 6: 315.

16. Humphrey AL and Hendrickson AE: Radial zones of high metabolic activity in squirrel monkey striate cortex. Soc Neurosci Abstr 1980, 6: 315.

17. Livingstone MS and Hubel DH: Anatomy and physiology of a color system in the primate visual cortex. J Neurosci 1984, 4: 309-56.

18. Zeki SM and Shipp S: The functional logic of cortical connections. Nature 1988, 335: 311-17.

19. Felleman DJ and Van Essen DC: Distributed hierarchical processing in the primate cerebral cortex. Cerebral Cortex 1991, 1: 1-47.

20. Hickey TL: The developing visual system. Trend Neurosci 1981, 4: 41-4.

21. Banks MS and Bennett TJ: Optical and photoreceptor immaturities limit the spatial and chromatic vision of human neonates. J Opt Soc Am A 1989, 5(12): 2059-79.

22. Atkinson $\mathbf{J}$ and Braddick OJ: The development course of cortical processing streams in the human infants. In Blakemore C (Ed). Vision: Coding and Efficiency. Cambridge University Press, 1990.

23. Garey L and de Courten C: Structural development of the lateral geniculate nucleus and visual cortex in monkey and man. Behavioural Brain Research 1983, 10: 3-15.

24. Slater A, Morison V, Somers M: Orientation discrimination and cortical function in the human newborn. Perception 1988, 17: 597-602.

25. Atkinson J, Hood B, Wattam-Bell J, Anker S, Tricklebank J: Development of orientation discrimination in infancy. Perception 1988, 17: 587-95.

26. Braddick OJ, Wattam-Bell J, Atkinson J: Orientation-specific cortical responses develop in early infancy. Nature 1986 320(6063): 617-19.

27. Wattam-Bell J: Analysis of infant visual evoked potentials (VEPs) by a phase-sensitive statistic. Perception 1985, 14: A33.

28. Braddick OJ, Atkinson J, Wattam-Bell J, Hood B: Characteristics of orientation-selective mechanisms in early infancy Invest Ophthalmol Vis Sci (Suppl) 1989, 30: 313.

29. Derrington AM and Lennie P: Spatial and temporal contrast sensitivities of neurons in lateral geniculate nucleus of macaque. Journal of Physiology 1984, 357: 219-40.

30. Wattam-Bell J: The development of motion-specific cortical responses in infants. Invest Ophthalmol Vis Sci (Suppl) 1988, 29: 24.

31. Wattam-Bell J: Displacement limits for the discrimination of motion direction in infancy. Invest Ophthalmol Vis Sci (Suppl) 1991, 32(4): 964.

32. Wattam-Bell J: The development of maximum velocity limits for direction discrimination in infancy. Perception 1990, 19(3): 369.

33. Aslin RN, Shea SL, Gallipeau JM: Motion thresholds in 3-month-old infants. Invest Ophthalmol Vis Sci (Suppl) 1988, 29: 26.

34. Atkinson J: Development of optokinetic nystagmus in the 
human infant and monkey infant: an analogue to development in kittens. In Freeman RD (Ed). NATO Advanced Study Institute Series. New York: Plenum Press, 1979.

35. Atkinson $\mathbf{J}$ and Braddick OJ: Development of optokinetic nystagmus in infants: an indicator of cortical binocularity: In Fisher DF, Monty RA and Senders JW (Eds). Eye Movements: Cognition and Visual Perception. Hillsdale NJ: Lawrence Erlbaum Associates, 1981.

36. Arterberry ME and Yonas A: Infants' sensitivity to kinetic information for three-dimensional object shape. Perception and Psychophysics 1988, 44: 1-6.

37. Yonas A: Infants' responses to optical information for collision. In Aslin RN, Alberts JR and Petersen MR (Eds). The Development of Perception: Psychobiological Perspectives. New York: Academic Press, 1981.

38. Atkinson J and Braddick OJ: Stereoscopic discrimination in infants. Perception 1976, 5: 29-38.

39. Braddick OJ, Atkinson J, Julesz B, Kropfl W, Bodis-Wollner I, Raab E: Cortical binocularity in infants. Nature 1980 , 288: $353-65$.

40. Petrig B, Julesz B, Kropfl W, Baumgartner G, Anliker M: Development of stereopsis and cortical binocularity in human infants: electrophysiological evidence. Science 1981, 213: 1402-5.

41. Braddick OJ, Wattam-Bell J, Day J, Atkinson J: The onset of binocular function in human infants. Human Neurobiology 1983, 2:65-9.

42. Fox R, Aslin RN, Shea SL, Dumais ST: Stereopsis in human infants. Science 1980, 207:232-324.

43. Held R, Birch EE, Gwiazda J: Stereoacuity of human infants. Proc Natl Acad Sci USA 1980, 77: 5572-4.

44. Braddick OJ and Atkinson J: Some recent findings on the development of human binocularity: a review. Behavioural Brain Research 1983,10: 141-50.

45. Braddick OJ and Atkinson J: Sensory selectivity attemtional control, and cross-channel integration in early visual development. In Yonas A (Ed). 20th Minnesota Symposium on Child Psychology. Hillsdale NJ: Lawrence Erlbaum Associates, 1988.

46. Wattam-Bell J, Braddick OJ, Atkinson J, Day J: Measures of infant binocularity in a group at a risk for strabismus. Clinical Vision Sciences 1987, 1(4): 327-36.

47. Smith J, Atkinson J, Braddick OJ, Wattam-Bell J: Development of sensitivity to binocular correlation and disparity in infancy. Perception 1988, 17: 395-6.

48. Atkinson J, Smith J, Anker S, Wattam-Bell J, Braddick OJ, Moore AT: Binocularity and amblyopia before and after strabismus surgery. Invest Ophthalmol Vis Sci (Suppl) 1991, 32(4): 964

49. Smith JC, Atkinson J, Anker S, Moore AT: A prospective study of binocularity and amblyopia in strabismus infants before and after corrective surgey: implications for the human critical period. Clinical Vision Sciences 1991, 6(5): 335-53.

50. Morrone MC, Burr DC, Fiorentini A: Development of contrast sensitivity and acuity of the infant colour system. Proc Roy Soc Lond B 1990, 242: 134-9.

51. Bower TG: Development in infancy. Freeman: San Francisco, 1984.

52. Baillargeon R, Spelke ES, Wasserman S: Object permanence in 5-month-old infants. Cognition 1987, 20: 191-208.

53. Mohn G and Van Hof-van Duin J: Development of the binocular and monocular visual fields of human infants during the first year of life. Clinical Vision Sciences 1983, 1: 51-64.

54. Heersema DJ, Van Hof-van Duin J, Hop WCJ: Age norms for visual field development in children aged 0 to 4 years using arc perimetry. Investigative Ophthalmology and Visual Science (Suppl) 1989, 30(3): 242.

55. Atkinson J, Hood B, Wattam-Bell J, Braddick OJ: Changes in infants' ability to switch visual attention in the first three months of life. Perception (In Press).

56. Julesz B: Textons, the elements of texture perception, and their interactions. Nature 1981, 290: 92-7.

57. Movshon JA, Thompson ID, Tolhurst DJ: Spatial summation in the receptive fields of simple cells in the cat's striate cortex. J Physiol 1978, 283: 53-77.

58. Pollen D and Ronner SF: Phase relationship between adjacent simple cells in the visual cortex. Science 1981, 212: 1409-11.

59. Braddick OJ, Atkinson J, Wattam-Bell J: Development of the discrimination of spatial phase in infancy. Vis Res 1986, 26(8): 1223-39.

60. Atkinson J, Wattam-Bell J, Braddick OJ: Infants' development of sensitivity to pattern 'textons'. Invest Ophthalmol Vis Sci (Suppl) 1986, 27: 265.

61. Beck J: Effect of orientation and shape similarity on perceptual grouping. Perception and Psychophysics 1966, 1: $300-2$.

62. Olson $\mathrm{R}$ and Attneave F: What variables produce similarity grouping? Am J Psycho 1970, 83: 1-21.

63. Nothdurft HC: Texton segregation by associated differences in global and local luminance distribution. Proc Roy Soc Lond B 1990, 239: 295-320.

64. Sagi D and Julesz B: 'Where' and 'what' in vision. Science 1985, 228: 1217-19.

65. Nothdurft HC: Sensitivity for structure gradient in texture discrimination. Vis Res 1985, 25: 157-68.

66. Braddick OJ and Atkinson J: Infants' and adults' segmentation of oriented textures. Invest Ophthalmol Vis Sci (Suppl) 1991, 32(4): 1045.

67. Atkinson $\mathrm{J}$ and Braddick OJ: Visual segmentation of oriented textures by infants. Behavioural Brain Research (In Press).

68. Humphrey NK: Vision in a monkey without striate cortex: a case study. Perception 1974, 3: 241-56.

69. Milewski AE: Infants' discrimination of internal and external pattern elements. J Exp Child Psychol 1976, 22: 229-46.

70. Bushnell IWR: Discrimination of faces by young infants Journal of Experimental Psychology 1982, 33: 298-30.

71. Weiskrantz L: Blindsight, A Case Study and Implications. Oxford: Clarendon Press, 1986.

72. Cowey A and Stoerig P: The neurobiology of blindsight. Trends in Neuroscience 1991, 14(4): 140-145.

73. de Renzi E: Oculomotor disturbance in hemispheric disease. In Johnston CW and Pirozzolo FJ (Eds). Neuropsychology on Eye Movements. Hillsdale NJ: Lawrence Erlbaum Associates, 1988.

74. Mountcastle VB: Brain mechanisms for directed attention. J Roy Soc Med 1978, 71: 14-28.

75. Schiller PH: The superior colliculus and visual function. In Darian-Smith I (Ed). Handbook of Physiology-The Nervous System III. Sensory Processes Part I. Bethesda MD: American Physiological Society, 1985. 